\title{
FIXED-POINT THEOREMS FOR ARCWISE CONNECTED CONTINUA ${ }^{1}$
}

\author{
G. S. YOUNG
}

L. E. Ward, Jr., recently proved in [4] a fixed-point theorem for certain arcwise connected spaces that generalizes a theorem of mineTheorem 2, below-and Borsuk's theorem [1] that an arcwise connected hereditarily unicoherent metric curve has the fixed-point property. His argument provides a proof of my result, but not of Borsuk's. That Borsuk's class of continua is contained in his follows from Borsuk's result only.

In this note I give a new sufficient condition for the fixed-point property that implies Borsuk's result, and that follows from my theorem and so from Ward's. I also give an example of an arcwise connected continuum that contains no simple closed curve but that does not have the fixed-point property, and prove a fixed-point theorem for a quite special class of contractible continua.

THEOREM 1. Let $M$ be an arcwise connected compact Hausdorff space that does not have the fixed-point property. Then $M$ contains either (1) $a$ continuum $N_{1}$ for which there is a map $f: N_{1} \rightarrow S^{1}$ which is onto and such that no closed proper subset of $N_{1}$ is mapped by $f$ onto $S^{1}$, and which is such that at most one point-inverse is nondegenerate, that one being connected; or (2) a continuum $N_{2}$ that contains a subset $R$ that is the oneto-one continuous image of a half-open interval and that is dense in $\mathrm{N}_{2}$, but that has no interior relative to $\mathrm{N}_{2}$; or (3) a continuum $N_{3}$ that is the union of a set $R$ that is the continuous one-to-one image of a half-open interval, and a continuum $B$, and for which there is a map $f: N_{3} \rightarrow K$, $K$ being the union of the circles $x^{2}+y^{2}=(2 / n) y, n=1,2,3, \cdots$, such that $f$ is one-to-one on $N_{3}-B$, such that $f(B)=(0,0)$, and such that no closed proper subset of $N_{3}$ is mapped by $f$ onto $K$.

We will see that this is a consequence of an earlier fixed-point theorem of the author's, proved in [5, p. 493]:

THEOREM 2. Let $M$ be an arcwise connected Hausdorff space which is such that every monotone increasing sequence of arcs is contained in an arc. Then $M$ has the fixed-point property.

Note that compactness is not required in Theorem 2. 1960.

Presented to the Society, January 29, 1960; received by the editors February 4,

${ }^{1}$ Part of the work on this paper was done under a grant by the National Science Foundation. 
Proof of Theorem 1. The proof is a straightforward analysis of the possible ways the hypothesis of Theorem 2 can fail in a compact space. Let $A_{1}, A_{2}, A_{3}, \cdots$ be a monotone increasing sequence of arcs that is not contained in an arc. Let $x$ be any non-end point of $A_{1}$. Then, for each $n, x$ divides $A_{n}$ into two $\operatorname{arcs} A_{n}^{\prime}$ and $A_{n}^{\prime \prime}$, the primes being chosen so that, for each $n, A_{n}^{\prime}$ is contained in $A_{n+1}^{\prime}$. If $M$ contains a simple closed curve, then that is a continuum of type 1 . Suppose that $M$ contains no simple closed curve. Then at least one of the two monotone increasing sequences $\left\{A_{n}^{\prime}\right\},\left\{A_{n}^{\prime \prime}\right\}$ does not lie in an arc. Hence there is no loss in assuming that $\left\{A_{n}\right\}$ itself is a sequence of arcs all having a common end point, $a$. There is also no loss in assuming that $A_{n+1}-A_{n}$ is never empty. Let $B$ denote the set lim sup $\mathrm{Cl}\left(A_{n+1}-A_{n}\right)$. An argument of a familiar type shows that $B$ is connected. For if $B$ were not, there exist two disjoint open sets $U$ and $V$, covering $B$, and each intersecting $B$. From some integer $k$ on, each set $\mathrm{Cl}\left(A_{n+1}-A_{n}\right)$ lies either in $U$ or in $V$. But $\mathrm{Cl}\left(A_{n+1}-A_{n}\right)$ and $\mathrm{Cl}\left(A_{n+2}-A_{n+1}\right)$ intersect. Induction shows that for $n>k$, either all the sets $\mathrm{Cl}\left(A_{n+1}-A_{n}\right)$ lie in $U$, or all lie in $V$. This gives a contradiction.

The set $\mathrm{U}_{n} A_{n}=R$ is the one-to-one continuous image of a halfopen interval. There are three possible relations between $R$ and $B$ : (1) The sets $R$ and $B$ are disjoint. Then there is an arc $x y$ from some point $x$ of $B$ to some point $y$ of $R$, such that $x=x y \cap B$ and $y=x y \cap R$. The point $y$ separates $R$ into two connected sets, $R^{\prime}$ and $R^{\prime \prime}$, where $R^{\prime \prime} \cup y$ is an arc from $a$ to $y$, and $R^{\prime} \cup y$ is again the one-to-one continuous image of a half-open interval. (In this case, it is actually a homeomorph of such an interval.) Let $N_{1}=x y \cup R^{\prime} \cup B$. The collection consisting of the set $B$ and of the individual points of $N_{1}-B$ is upper semi-continuous and defines a map $f: N_{1} \rightarrow S^{1}$ satisfying the conditions of part (1) of the conclusion of the theorem. (2) The sets $R$ and $B$ intersect, but some arc $a u$ of $R$ contains $R \cap B$. (It may actually happen that $R \cap B$ consists of just two points.) Let $R^{\prime}$ be the set $R-a u$, and let $N_{1}=R^{\prime} \cup B$. Then in the same way as above, we have the desired map $f: N_{1} \rightarrow S^{1}$. (3) There is an integer $k$ such that $\bigcup_{n=k}^{\infty}\left(A_{n+1}-A_{n}\right)=R^{\prime}$ is contained in $B$. Then $N_{2}=B$ is the desired continuum of the second part of the conclusion, with $R^{\prime}=R$. (4) No arc of $R$ contains $R \cap B$, and also there is no integer $k$ such that $\bigcup_{n=k}^{\infty}\left(A_{n+1}-A_{n}\right)$ is contained in $B$. In this case, $\cup A_{n}-B$ is the union of a countable number of disjoint open intervals, $I_{1}, I_{2}, I_{3}, \cdots$. Let $N_{3}=B \cup \cup I_{n}$. The upper-semicontinuous collection consisting of $B$ and of the individual points of the intervals $\left\{I_{n}\right\}$ defines a map of $N_{3}$ onto a continuum of the third type of the conclusion of Theorem 1 , satisfying the desired conditions. 
From Theorem 1, we get an easy proof of Borsuk's theorem.

THEOREM 3. If $M$ is an arcwise connected, hereditarily unicoherent metric [or Hausdorff] curve, then $M$ has the fixed-point property.

Proof. Note that a continuum of either of the first or third types described in Theorem 1 is not unicoherent, so that $M$ contains neither of these. Next, a hereditarily unicoherent arcwise connected continuum $M$ contains no indecomposable subcontinuum. For suppose that $S$ is such an indecomposable subcontinuum of $M$. There is an arc $A$ in $M$ whose end points lie in different composants of $M$. Then $A$ is not a subset of $M$, and $A \cup S$ is not unicoherent. Theorem 3 follows then from the next result, which seems to have escaped publication, and which shows that $M$ can contain no continuum of the second type.

THEOREM 4. If a hereditarily unicoherent continuum $S$ contains a dense subset $R$ that is the one-to-one continuous image of a half-open interval, but that contains no interior points, then $S$ is indecomposable.

Proof. Suppose that $S$ is the union of two proper subcontinua, $A$ and $B$. Each has an interior, Int $A=S-B$ and Int $B=S-A$, relative to $S$. We may order the points of $R$ by their order in the halfopen interval, the image of the end point being the first point of $R$. Let $a_{1}$ be a point of $R \cap \operatorname{Int} A, b$ be a point of $R \cap \operatorname{Int} B$ that follows $a_{1}$ in $R$, and $a_{2}$ be a point of $R \cap \operatorname{Int} A$ that follows $b$ in $R$. Then if $a_{1} a_{2}$ denotes the arc of $R$ from $a_{1}$ to $a_{2}, A \cup a_{1} a_{2}$ is not unicoherent.

The join, in the sense of combinatorial topology, of a Cantor set and a point contains a subset $R$ that is the continuous image of a half-open interval, that is dense in the join, and that has no interior, showing that the one-to-one property is required.

Theorem 1 does not imply Theorem 2. In fact, for each integer $n>1$, there is an arcwise connected, contractible and metric continuum containing no subcontinuum of any of the three types of Theorem 1. Let $X$ be a continuum of dimension $n-1$ that contains no arc; for example, the product of $n-1$ pseudo-arcs [3]. Let $M$ be the join of $X$ and a point $p$. If $S$ is a continuum in $M$ of one of the three types, $S-p$ cannot lie in one interval of the join, and the projection of $M-p$ onto $X$ will map some arc of $S-p$ onto a nondegenerate continuum in $X$. However, Borsuk's hypothesis cannot hold in $X$, since a continuum of dimension greater than one cannot be hereditarily unicoherent. We can modify the example slightly, by replacing $M$ by two such joins, having in common only one point, on the base of each, and show that for each integer $n>1$, there is an arcwise connected and noncontractible metric continuum containing no subcontinuum of any of the three types of Theorem 1. 
Kinoshita gave an example [2] of a contractible continuum that has no fixed point. Since it contains a 2-cell, it contains continua of all the types of Theorem 1. That result, however, does imply one fixed-point theorem for contractible continua.

Theorem 5. If $M$ is a contractible Hausdorff continuum such that each two points are the end points of only one arc, then $M$ has the fixedpoint property.

Proof. Suppose that $M$ contains a continuum $N$ satisfying condition (1) of Theorem 1. The uniqueness of arcs shows that $N$ cannot be a simple closed curve, so that one point-inverse under the mapping $f$ of that condition is a nondegenerate continuum, $B$. The proof of part (1) of Theorem 1 shows that we can assume that $N$ is the union of $B$ and the continuous one-to-one image $R$ of a half-open interval, $R \cap B$ consisting of the image of the end-point of that interval. Let $c: M \times I \rightarrow M$ be a contraction, satisfying $c(x, 1)=p$. By uniform continuity of $c$, for each positive number $\epsilon$, there is a positive number $\delta$ such that if $d(x, y)<\delta$, then for all $t$ in $I, d[c(x, t), c(y, t)]$ $<\epsilon$.

Let $y$ be a point of $B$ not in $R$ and not $p$. The set $c(y \times I) \cap R$ may be empty, but if not, it is connected. For if $c(y \times I) \cap R=H \cup K$, separated, then there exists an arc $A_{1}$ in $R$ from a point $h$ in $H$ to a point $k$ in $K$ and there is an arc $A_{2}$ in $c(y \times I)$ from $h$ to $K$, and $A_{1} \cup A_{2}$ contains a simple closed curve. If $e$ denotes the end point of $R$, which is in $B$, it is conceivable that $e$ is not in $c(y \times I)$. It is not possible, however, that for some point $x$ in $R, c(y \times I)$ contains the set $R_{x}$ consisting of all the points $z$ in $R$ such that $x$ is on the arc $e z$ of $R$. For suppose that this occurred. Then $c(y \times I)$ contains $B$. Let $U$ be a relatively open connected subset of the Peano continuum $c(y \times I)$ that contains $e$ (which is in $N$ ), but does not contain $x$. Let $x^{\prime}$ be a point of $R_{x} \cap U$. There is an arc $x^{\prime} e$ in $U$, and in $R$ there are arcs $e x, x x^{\prime}$. The union $x^{\prime} e \cup e x \cup x x^{\prime}$ contains a simple closed curve, which is impossible. We can thus conclude that $R-c(y \times I)$ contains a set $R_{x}-x$, for some $x$ in $R ; x$ will be in $c(y \times I)$. If $z$ is a point of $R_{x}$, then $c(z \times I)$ contains the arc $x z$ of $R$; otherwise $x z \cup c(z \times I) \cup c(y \times I)$ contains a simple closed curve.

Now let $\epsilon_{1}, \epsilon_{2}, \epsilon_{3}, \ldots$ be a sequence of positive numbers approaching 0 . For each $\epsilon_{n}$, let $\delta_{n}$ be the corresponding number $\delta$ defined in the last sentence of the first paragraph of this proof, and let $x_{n}$ be a point of $R_{x}$ within $\delta_{n}$ of $y$. Then $d\left[c\left(x_{n}, t\right), c\left(y_{0}, t\right)\right]<\epsilon_{n}$ for all $t$ in $I$. Let $z$ be a fixed point of $R_{x}$; there is no loss in supposing that $z$ is in each $\operatorname{arc} x x_{n}$ in $R$. Then by our last paragraph, $z$ is in each set $c\left(x_{n} \times I\right)$. For each $\epsilon_{n}$, then, $d(z, c(y \times I))<\epsilon_{n}$, so that $z$ belongs to the 
set $c(y \times I)$. But this is a contradiction.

Modifications of this argument dispose of each of the other two cases.

Either from Theorem 5 or, quicker, from Borsuk's theorem, it follows that a one-dimensional contractible continuum $C$ has the fixed-point property, since every subcontinuum is homologically acyclic, so that $C$ contains no simple closed curve.

Let $C_{1}$ be a continuum in the lower half $x y$-plane joining the point $(2,0,0)$ to the interval $[-3,-1]$ of the $x$-axis, $C_{1}$ being homeomorphic to the closure of the graph of $y=\sin 1 / x, 0<x \leqq \pi$, with the interval $[-3,-1]$ corresponding to the limiting interval of the graph. Let $C_{2}$ be the image of $C_{1}$ under the rotation of the $x y$-plane about the origin through an angle of $\pi$. Let $L_{1}$ and $L_{2}$ be straightline intervals joining $(2,0,0)$ and $(-2,0,0)$ to $(0,0,1)$. Let $R$ be a set homeomorphic to a half-open interval that (1) has only $(0,0,1)$ in common with $C_{1} \cup C_{2} \cup L_{1} \cup L_{2}$ and (2) "spirals down" to $C_{1} \cup C_{2}$ in such a way that (a) there is a sequence of $\operatorname{arcs} X_{1}, X_{2}, X_{3}, \cdots$ filling up $R$ such that $X_{i} \cap X_{j}$ is empty for $j \neq i+1, i-1$, and is an end point of each for $j=i+1, i-1$, and (b) $C_{1}=\lim X_{2 j}$ and $C_{2}=\lim X_{2 j+1}$. Let $M=C_{1} \cup C_{2} \cup L_{1} \cup L_{2} \cup R$. Then $M$ is arcwise connected by unique arcs, and is compact. We define a continuous map $f: M \rightarrow M$ that has no fixed point. Let $f_{1}: M \rightarrow M$ be a map that on $C_{1} \cup C_{2} \cup L_{1} \cup L_{2}$ is the rotation of $E^{3}$ about the $Z$-axis through an angle of $\pi$, and that on $R$ is the identity; $f_{1}$ is not continuous. Let $f_{2}: M \rightarrow M$ be a map that is a homeomorphism on $R$ and maps each arc $X_{n}$ onto $X_{n+1}$; that is the identity on $C_{1} \cup C_{2}$, and that maps each set $L_{j}, j=1$, 2, homeomorphically onto $L_{j} \cup X_{1}$, the points $(2,0,0)$ and $(-2,0,0)$ being kept fixed; $f_{2}$ is not continuous either. The composition $f=f_{2} f_{1}$, however, is continuous, and no point is left fixed.

I have no such example in the plane, nor do I have a continuum $M$ that does not have the fixed-point property for homeomorphisms.

\section{BIBLIOGRAPHY}

1. K. Borsuk, $A$ theorem on fixed points, Bull. Acad. Polon. Sci. Cl. III vol. 2 (1954) pp. 17-20.

2. Shin'ichi Kinoshita, On some contractible continua without fixed-point property, Fund. Math. vol. 40 (1953) pp. 96-98.

3. E. E. Moise, An indecomposable plane continuum which is homeomorphic to each of its nondegenerate subcontinua, Trans. Amer. Math. Soc. vol. 63 (1948) pp. 581-594.

4. L. E. Ward, Jr., A fixed point theorem for chained spaces, Pacific J. Math. vol. 9 (1959) pp. 1273-1278.

5. G. S. Young, The introduction of local connectivity by change of topology, Amer. J. Math. vol. 68 (1946) pp. 479-494.

TUlane UNIVERSITY 